\title{
TRADICIÓN Y NUEVAS PERSPECTIVAS EN EL ESTUDIO DEL COMPUESTO NOMINAL ALEMÁN
}

\author{
ULRIKE OSTER \\ Universitat Jaume I, Castelló \\ oster@trad.uji.es
}

\section{RESUMEN}

Este artículo tiene como objetivo reivindicar la utilidad de seguir profundizando en la investigación lingüística en el campo de la composición nominal del alemán. Con el fin de detectar aspectos concretos que merecerían un estudio más detallado, daremos primero una visión general y resumida de las distintas perspectivas teóricas de la lingüística desde las cuales se ha tratado este fenómeno. A continuación, tratamos algunos enfoques más específicos, en los que los aspectos pragmáticos, textuales y cognitivos desempeñan un papel relevante. Esta revisión nos lleva a identificar posibles nuevas vías de investigación, especialmente en lo que se refiere a los lenguajes especializados, el aspecto textual y/o la comparación con otras lenguas.

PALABRAS CLAVE: Formación de palabras; composición nominal

\section{ABSTRACT}

This article argues that nominal compounding in German, in spite of the wealth of publications dedicated to it, still remains an interesting subject for linguistic analysis from various points of view. The paper sets out with a general overview of the contributions of different theoretical linguistic perspectives to the description of compounding, followed by an account of some specific approaches that address pragmatic, textual, and cognitive aspects. This leads us to identify several possibilities for further research, es- 
pecially with regard to specialised languages, textual aspects, and/or the contrast with other languages.

KEY WORDS: Word-formation; nominal compounds

\section{INTRODUCCION}

La formación de palabras $-\mathrm{y}$, dentro de ésta, sobre todo la composición nominal- es un tema que ha suscitado mucho interés por parte de los lingüistas, especialmente en el ámbito de la filología alemana. En ocasiones, incluso se considera que sobre los compuestos nominales alemanes está todo dicho. A pesar de la existencia de numerosos estudios sobre el tema, sin embargo, la composición es un fenómeno tan rico en matices que puede dar lugar a investigaciones que profundicen en diversos aspectos. Por ello, en este artículo nos hemos propuesto descubrir dónde quedan zonas blancas en la geografía tan explorada de la composición nominal alemana, partiendo de una revisión de la investigación existente al respecto.

\section{PANORAMA HISTORICO}

Comenzaremos por un breve itinerario por las principales tendencias que, bajo la influencia de los cambios de perspectiva dentro de la lingüística general, se han ido sucediendo (aunque no de forma completamente lineal, sino con solapamientos) en la investigación sobre la formación de palabras del alemán. ${ }^{1}$ En la gramática tradicional histórico-filológica, y hasta la primera mitad del siglo XX, el interés por la formación de palabras era sobre todo de tipo histórico, es decir, se concentraba en el papel de la formación de palabras en la evolución del vocabulario de la lengua. Encontramos las primeras referencias a las palabras compuestas en las gramáticas del alto alemán moderno, que datan del siglo XVI. Algún tiempo después, las gramáticas elaboradas en los siglos XVII y XVIII ya conceden un espacio considerable a este tema (por ejemplo, Adelung, 1793-1801). En el siglo XIX, las grandes gramáticas históricas del alemán (Grimm, 1826; 1831; Wilmanns, 1896) dedican voluminosos tomos a la formación de palabras, centrándose, sobre todo, en aspectos etimológicos y contrastivos. Con la llegada del siglo XX, en cambio, se produce un cierto cambio de perspectiva. Paul (1896, 1903, 1920), por ejemplo, centra su análisis en el alemán contemporáneo, reivindicando una descripción tanto formal como semántica. Estas obras tempranas de la descripción de la formación de palabras en el nivel morfológico y etimológico proporcionan la base sobre la que se asentarán estudios posteriores que explorarán otros aspectos del tema.

${ }^{1}$ Al tratar la composición nominal del alemán, nuestro interés se centra, sobre todo, en obras que analizan esta lengua. No obstante, no dejaremos de mencionar a autores que, aunque refiriéndose a una lengua distinta del alemán, hayan hecho una contribución teórica o metodológica importante para la evolución de la investigación en este campo. 
Frente a las descripciones morfológicas de la gramática tradicional, centradas en la vertiente formal de los compuestos, lingüistas estructuralistas como Coseriu $(1968,1977)$ o Gauger (1971) abogan por un tratamiento referido al significado. En lo que respecta a la distinción establecida por Dokulil (1968) entre Wortbildung (el proceso de formación de la palabra) y Wortgebildetheit (el producto de dicho proceso), la opción de los investigadores estructuralistas es clara: su objeto de estudio es el producto, la palabra creada. De la combinación de los dos puntos de vista mencionados, la perspectiva formal de la gramática tradicional y la del significado de la semántica estructural, han surgido las descripciones más amplias de la formación de palabras del alemán. Entre éstas están, por ejemplo, Henzen (1957) y sobre todo las obras de Fleischer (1969-1995) y Erben (1975-2000), ambas en sucesivas ediciones, así como las publicaciones que fueron fruto de un proyecto de investigación sobre la composición alemana, dirigido por Erben. Entre ellas, Ortner y Ortner (1984) y Ortner, et al. (1991) describen específicamente los compuestos nominales.

En cambio, los investigadores que se encuadran dentro de la gramática generativa se interesan principalmente por el proceso de creación de nuevas palabras. Una vez establecida la teoría generativista sobre la estructura y organización de la gramática, sus principios no tardaron en ser aplicados a la formación de palabras. Así, encontramos a Marchand (1960) y a Lees (1960) para el inglés, así como a Motsch (1970) y —ya combinado con aspectos semánticos derivados de la gramática de casos de Fillmore - a Kürschner (1974), para el alemán.

Un problema crucial debatido entre los lingüistas pertenecientes a este paradigma es la pregunta de si la formación de palabras pertenece a la sintaxis, al lexicón o si conforma un módulo independiente. La adopción de una u otra solución a este problema ha dado lugar a perspectiva distintas:

- La corriente transformacionalista trata de hallar reglas para la formación de palabras que permitan predecir la formación de palabras compuestas. Los compuestos son explicados como la transformación de oraciones base de la estructura profunda en manifestaciones superficiales de la lengua.

- La corriente lexicalista, en cambio, propugna la inclusión de las palabras compuestas en el lexicón (Jackendoff, 1975; Aronoff, 1976, y —para el alemán y con matices- Toman, 1983; Olsen, 1986). Según esta concepción, los compuestos están almacenados en el lexicón, junto con sus características y reglas específicas, así como con reglas de redundancia para evitar el almacenamiento múltiple de información repetida.

En resumen, los planteamientos generativistas tienen en común el objetivo de hallar las reglas que permiten la generación de las palabras existentes o potenciales de una lengua.

\section{ESTUDIOS SOBRE ASPECTOS ESPECÍFICOS DE LA COMPOSICIÓN NOMINAL}

La desviación del interés de la investigación lingüística hacia aspectos pragmáticos y comunicativos, a partir de los años sesenta y setenta, también se produce en el campo de la investigación sobre compuestos. En este apartado presentaremos brevemente algunos resultados referidos a aspectos pragmáticos, textuales y cognitivos de la composición 
nominal, así como estudios que tratan este tema desde un punto de vista contrastivo y/o dentro de los lenguajes especializados.

\subsection{Enfoques pragmáticos}

A partir de los años setenta surgen los primeros trabajos interesados en la vertiente pragmática de la formación de palabras. A través de experimentos psicolingüísticos, Downing (1977) llega a la conclusión de que no existen restricciones semánticas para la creación de compuestos nominales en ausencia de contexto. En cambio, la aceptabilidad del compuesto depende de condiciones pragmáticas como la situación en la que se usa, la función que cumple o la interpretabilidad del compuesto en ese contexto por medio del contenido informativo de los constituyentes.

Carroll y Tanenhaus (1975), a su vez, son los primeros en formular principios pragmáticos para la creación de compuestos. Según estos autores, la formación de palabras obedece a dos principios funcionales: «la hipótesis del vacío semántico», por una parte, según la cual la existencia de un concepto que carece de denominación puede provocar la creación de un neologismo y, por otra, el «principio minimax», que predice la tendencia del hablante a minimizar esfuerzos al tiempo que intenta maximizar la información trasmitida (Carroll y Tanenhaus, 1975: 51). Bauer (1979) va más lejos y especifica en qué consiste la información pragmática que interviene en la producción y recepción de los compuestos: el conocimiento y las creencias sobre el mundo y la sociedad, así como el contexto en el que aparece el compuesto.

En el ámbito del alemán, un proyecto de investigación sobre formación de palabras ${ }^{2}$ se propone estudiar las condiciones comunicativas y semántico-pragmáticas de la producción, la utilización y la comprensión de los compuestos nominales alemanes. Para comprobar sus hipótesis, el grupo realiza una serie de experimentos sobre la producción y la recepción de compuestos (tiempos de recepción, parafraseado, evaluación de la aceptabilidad de compuestos y paráfrasis) (Brekle, 1983-1985: Arbeitsberichte 19, 24-28, 30-37). De esta forma, llegan a identificar principios pragmáticos para la producción de los compuestos (Stöhr, 1984a), procesos que guían la interpretación y que marcan y estructuran el texto (Wildgen, 1982), así como una clasificación de los compuestos basada en condiciones pragmáticas (Stöhr, 1984b).

\subsection{Enfoques textuales}

Una de las facetas resaltadas por Downing (1977) y Bauer (1979) — la vertiente contextual de los compuestos- se convertirá en centro de atención del interés de una serie de investigadores que analizan las funciones desempeñadas por los compuestos en el nivel textual: ${ }^{3}$

${ }^{2}$ El título completo del proyecto, dirigido por Brekle entre 1980 y 1984, es «Kommunikative und pragmatisch-semantische Bedingungen der Aktualgenese, der Verwendung und des Verstehens von Nominalkomposita».

${ }^{3}$ Un estado de la cuestión reciente se encuentra en Kauffer (2005). 
- Wladowa (1975) y Schröder $(1978,1983,1985)$ analizan la interrelación de los compuestos con el texto y les atribuyen un papel cohesivo (textverflechtende Funktion).

- Para los textos literarios, Dressler (1981) y Betten (1987) inciden en la función estilística de los compuestos novedosos. Según Betten (1987: 84), su importancia reside en actuar como pilares de la estructura textual global, ya que vehiculan la expresión de las reflexiones del autor y sostienen y determinan el edificio conceptual que éste va construyendo.

- Peschel (2002) resume las funciones textuales de la formación de palabras como sigue: su contribución a la progresión temática, la consolidación conceptual, los juegos de palabras y el efecto leitmotiv (en los textos literarios).

- Basándose en el análisis de textos técnicos (patentes), Dederding $(1982,1983)$ sostiene que las funciones textuales del compuesto consisten en asegurar la exactitud de la referencia y en incrementar la redundancia (vid. el apartado 3.4).

- Matussek (1994), por su parte, analiza el papel de los compuestos novedosos llamativos en un corpus de textos periodísticos y llega a la conclusión de que cumplen una función valoradora. El grado de llamatividad depende, en primer lugar, de la medida en que el contexto apoya o contradice el significado presumible de un lexema complejo (Matussek, 1994: 38).

Estas propuestas nos parecen complementarias —más que contradictorias - en tanto que, al haberse adoptado un método de análisis de corpus de géneros concretos, los resultados reflejan necesariamente la especificidad del género en cuestión. Bajo esta óptica, la función dominante hallada para cada género parece muy coherente con las características específicas que se les suelen atribuir. De esta forma, obtenemos diversas funciones que tienen distintos grados de relevancia para cada género:

\begin{tabular}{|l|l|}
\hline Género & Función dominante de los compuestos \\
\hline Textos literarios & Función estilística \\
\hline Textos periodísticos & Función valorativa \\
\hline Patentes & $\begin{array}{l}\text { Función de asegurar la exactitud de la referencia e incrementar } \\
\text { la redundancia }\end{array}$ \\
\hline Sin restricción de géneros & Función cohesiva y de progresión temática \\
\hline
\end{tabular}

Tabla 1. Género textual y función dominante de los compuestos.

\subsection{Enfoques cognitivos}

Una vez estudiada la vertiente pragmática y textual de la producción de los compuestos, empieza a surgir la pregunta de cuáles son los mecanismos cognitivos que permiten la interpretación de una palabra compuesta. 
Significado potencial y significado textual

Un primer aspecto que parece relevante para ello es la distinción, establecida independientemente por Rickheit (1993) y Matussek (1994), entre el significado que una palabra compuesta podría tener fuera de contexto (el significado potencial) y el significado real que posee en la situación en la que es utilizada (el significado contextual). En el modelo de representación semántica de Rickheit (1993), el significado textual (llamado aquí Referenzkonzept) de un compuesto no solamente es actualizado por el contexto. Los dos conceptos léxicos (Wortkonzepte) combinados en un compuesto se actualizan mutuamente, puesto que cada uno de ellos pertenece a una categoría determinada. Ello determina a su vez el tipo de atributos que puede tener y las relaciones canónicas que son posibles entre ambos. Según Rickheit, enfocamos aquel aspecto del significado y aquellos atributos que permiten que ambos conceptos léxicos sean compatibles para la interpretación del conjunto.

\section{Tipos de conocimientos}

Una segunda cuestión de gran relevancia para el nivel cognitivo son los tipos de conocimiento que intervienen en la comprensión de compuestos. Heringer (1984a; 1984b) distingue entre dos tipos:

- el conocimiento permanente o genérico (lingüístico y enciclopédico), y

- el conocimiento actualizado (Laufwissen), formado tanto por el conocimiento del contexto y de la situación comunicativa, como por el conocimiento episódico compartido por hablante y oyente.

Para interpretar un compuesto fuera de contexto —es decir, para determinar su significado potencial— recurrimos al conocimiento genérico. Pero si el compuesto es utilizado en una situación determinada, los demás tipos de conocimiento pueden provocar que se active una interpretación diferente, correspondiente a su significado contextual. Para comprender lo que Heringer entiende por activación, debemos tener en cuenta que concibe los lexemas como nodos de una red semántica que están relacionados más o menos estrechamente con otros lexemas. Activación significa, pues, que se producen asociaciones mentales, basadas en regularidades que el hablante ha ido construyendo a lo largo de su «historia comunicativa» (Heringer, 1984b: 6).

\section{Mecanismos cognitivos de la comprensión de compuestos}

Establecida la diferencia entre significado potencial y significado contextual, desde la lingüística cognitiva se han intentado describir los mecanismos cognitivos que intervienen en el proceso de comprensión de los compuestos. Resumiremos a continuación las propuestas de Ryder (1994), por una parte, y la de Turner y Fauconnier (1995), por otra. 
a) Un modelo basado en la teoría de los esquemas

Ryder (1994) propone un modelo de la comprensión de compuestos novedosos basado en la teoría de los esquemas (en el sentido de Anderson y Pearson, 1988). Para interpretar una palabra compuesta, según Ryder, necesitamos encontrar un esquema que ambos constituyentes del compuesto compartan. Para explicar este principio, la autora recurre a una sencilla oración ("The boy walked"), en la que el esquema de 'walk' necesariamente tiene un nodo que debe ser rellenado por una entidad que lleva a cabo la acción. 'Boy', como entidad animada, puede desempeñar ese papel, debido a que es uno de los esquemas en los que encaja. Cuando no hay un esquema común a ambos conceptos, como en la oración "The fish walked", el oyente tiene que adaptar uno o ambos esquemas para hacerlos compatibles, por ejemplo imaginando un pez mutante con patas. En el caso de la combinación de dos sustantivos en un compuesto, ambos suelen contener un gran número de esquemas, por lo cual es frecuente que haya uno o más esquemas compartidos. Un ejemplo extremo sería el de Heringer (1984b) en el que podemos explicar los distintos significados potenciales de Fischfrau ('mujer que vende pescado', 'mujer piscis', 'mujer del pez', 'mujer y pez = sirena', 'mujer que es fría como un pez', etc.) por el hecho de que Fisch y Frau comparten muchos esquemas.

\section{b) Los espacios mentales combinados}

Recogiendo las ideas de Fauconnier (1985), Turner y Fauconnier (1995: 184) definen los espacios mentales como «paquetes» conceptuales dinámicos que se construyen y modifican durante la comunicación. Uno de los mecanismos cognitivos más importantes en el proceso de construcción de espacios mentales es la integración conceptual (conceptual integration o blending), un ejemplo de la cual es la composición nominal. Según esta visión, en un compuesto se combinan dos espacios mentales. En el compuesto inglés land yacht ( $\rightarrow$ 'yate terrestre' $\rightarrow$ 'coche de lujo'), por ejemplo, se abren los espacios mentales 'yate' y 'tierra', que hacen referencia a dos dominios distintos. Estos espacios representan la estructura parcial relevante de un dominio (Fauconnier y Turner, 1994: 5). A partir de aquí, el receptor tiene que realizar una proyección entre ambos espacios mentales. En esta proyección, 'agua' se corresponde con 'tierra', el 'capitán' con el 'conductor' y el 'yate' con un 'coche'. En el espacio mental combinado, el dueño del coche/barco es una persona rica y el vehículo (al que hace referencia el compuesto en cuestión) es un coche de lujo.

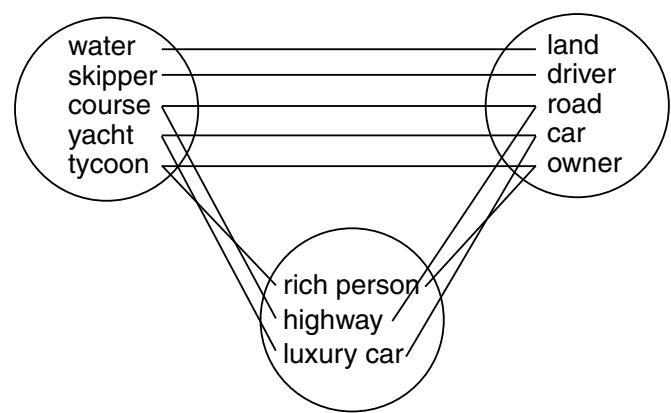

Ilustración 1: La combinación de los espacios 'land' y 'yacht' (Turner y Fauconnier 1995: 195). 
No obstante, no se trata de la combinación exacta de dos espacios. Hay elementos de ambos espacios que no se tienen en cuenta para la confección del espacio combinado (la tripulación del barco, etc.) y, por otra parte, éste contiene elementos específicos que no provienen de ninguno de los espacios de entrada (elevalunas eléctricos, etc.) (Turner y Fauconnier, 1995: 196).

La concepción de Turner y Fauconnier (1995) muestra un gran parecido con el enfoque basado en los esquemas. Ambos entienden que la persona que interpreta un compuesto busca una faceta en ambos conceptos combinados que los hace compatibles. ${ }^{4}$ Lo que es novedoso del modelo de los espacios mentales combinados, sin embargo, es que tiene en cuenta que el espacio resultante contiene elementos nuevos que no proceden de ninguno de los espacios de partida y que la construcción de dicho espacio resultante es un proceso creativo.

\subsection{Los compuestos en textos especializados}

Otro aspecto específico que merece mención aparte es la composición nominal en textos especializados, especialmente en textos técnicos. Ello está relacionado, sin duda, con la gran importancia numérica de las palabras compuestas entre los términos técnicos ${ }^{5}$. Aunque en todos estos estudios la descripción morfológica ocupa un papel importante, nos centraremos en el aspecto funcional, puesto que es aquí donde se detecta una clara diferencia respecto a los compuestos en textos generales.

Como hemos mencionado en el apartado 3.2., Dederding (1982, 1983) describe cómo los compuestos en textos especializados cumplen sobre todo la función de especificar la referencia. En un corpus de textos de patentes, este autor comprueba que, contrariamente a los postulados de la teoría general de la terminología y de las normas para la redacción de este tipo de textos, distintos compuestos nominales pueden designar un único objeto. Por ejemplo, en un texto son utilizados como términos equivalentes las siguientes unidades léxicas: Dampfrohr, Düsenrohr, Dampfführungsrohr y Dampf-Düsenrohr (Dederding, 1982: 155). El análisis de los casos de sinonimia le lleva a constatar que hay una variación denominativa relativamente alta, sobre todo en lo que respecta al primer constituyente. Concluye que la creación y utilización de los compuestos obedece a dos principios contrapuestos: el de la relativa libertad para crear palabras («Prinzip relativer wortbildnerischer Freiheit») y el de la necesidad de una diferenciación suficiente frente a otros elementos del contexto («Prinzip der Notwendigkeit hinreichender Differenzierung von Kontextelementen») (Dederding, 1982: 159).

En un estudio posterior, Zhu $(1987,1990)$ realiza una distinción funcional de los compuestos técnicos en el marco de un estudio descriptivo de la composición nominal

\footnotetext{
${ }^{4}$ Este paralelismo es aún más claro en Fauconnier y Turner (1994), donde los autores añaden un cuarto espacio, el espacio genérico. Este es un espacio abstracto que refleja los papeles, marcos y esquemas compartidos por los espacios de partida (Fauconnier y Turner, 1994: 5).

5 Por ejemplo, Herzog (1976: 75) habla de un 68\% de compuestos entre los términos alemanes de informática, e Ischreyt (1965: 177) contabiliza un $85 \%$ en su recuento de las unidades terminológicas encontradas en normas alemanas.
} 
alemana en el ámbito temático de la técnica de silicatos. Distingue entre dos tipos de compuestos técnicos:

a) Los compuestos con función denominativa, cuya motivación es la de dar nombre a objetos o hechos especializados. Una vez formados y aceptados, se convierten en términos lexicalizados. Como tales, hacen referencia a un concepto claramente definido que puede ser mucho más específico que lo que sugiere la combinación de los constituyentes. La fuente principal de conocimiento para una interpretación correcta de los compuestos técnicos es el conocimiento especializado (Zhu, 1987: 269).

b) Los compuestos con función textual son combinaciones efímeras cuya forma está supeditada a la construcción textual, aunque su significado está también estrechamente relacionado con fenómenos técnicos (Zhu, 1987: 271).

Esta distinción parece muy relevante para el análisis de compuestos técnicos y, al igual que en el nivel general se han detectado funciones diferentes de los compuestos, sería interesante concretar en qué consisten las funciones de construcción textual de los compuestos especializados.

\subsection{Estudios contrastivos}

Una consecuencia lógica del interés suscitado por la composición nominal del alemán es que una serie de autores haya comparado el sistema de composición del alemán con los sistemas de formación de palabras de otras lenguas. Este contraste interlingüístico lo realizan, por ejemplo, Cartagena y Gauger (1989) entre el alemán y el español y Wolf (1990) entre el alemán y el francés. Por otra parte, una serie de autores se ha dedicado a realizar una comparación funcional entre los compuestos nominales alemanes y las estructuras equivalentes en otras lenguas. Veremos más en detalle algunas de estas propuestas. Realizan, respectivamente, una comparación morfológica (Angele, 1992), funcional y textual (Gómez, 2001) y de la motivación semántica (Shaw, 1979).

Angele (1992) estudia las equivalencias españolas de compuestos nominales alemanes hallados en un corpus compuesto por textos alemanes y sus respectivas traducciones al español. De los resultados cuantitativos de su análisis morfológico cabe destacar el predominio, en las equivalencias españolas, de las construcciones adjetivales $(31,9 \%)$, las construcciones con la preposición de $(35,8 \%)$ así como, en menor medida, los monolexemas $(14,9 \%)$. Estos tres tipos de construcción abarcan en conjunto más del $80 \%$ de las equivalencias. Por otra parte, Angele (1992: 198) concluye que los factores más importantes que influyen en la elección del equivalente español son el tipo de relación semántica y el contexto. El análisis de las relaciones semánticas internas de los compuestos es sin duda interesante con vistas a su traducción, pero pensamos que no podemos presuponer — como parece hacer Angele - que la equivalencia en la otra lengua presentará el mismo tipo de relación.

También Gómez (2001) estudia el compuesto nominal alemán con vistas a su traducción al español. Su primer objetivo consiste en «sistematizar [...] las características 
tanto micro como macroestructurales del $\mathrm{CNa}$ [compuesto nominal alemán] en textos generales», dando cuenta de «[...] las convergencias y divergencias que presentan el sistema compositivo alemán y el sistema atributivo español.» (Gómez, 2001: 5). La autora basa esta sistematización en el análisis de un corpus de textos periodísticos alemanes de una misma temática y sus respectivas traducciones al español. En cuanto a la comparación morfológica, sus resultados cuantitativos confirman los datos hallados por Angele (1992). Lo más destacable de este trabajo es, sin embargo, el contraste de las formas encontradas en ambas versiones del corpus desde el punto de vista funcional y textual. En cuanto a la funcionalidad de las formas comparadas, Gómez explica el alto grado de coincidencia del compuesto nominal alemán con la estructura nombre más adjetivo en español por el hecho de que comparten dos características importantes: ambas formas constituyen «un importante recurso de economía de material lingüístico» (Gómez, 2001: 270) y se caracterizan por la indeterminación de la relación semántica entre sus constituyentes.

Desde el punto de vista textual, cabe destacar varias de sus conclusiones:

- En cuanto a la utilización, por parte de las dos lenguas, de medios distintos para establecer la progresión textual, concluye que:

En alemán la progresión textual queda asegurada mediante la repetición total o parcial de los constituyentes del compuesto, mientras que el español se sirve para desempeñar esa misma función textual de una estructura sintáctica que le permite introducir artículos anafóricos o catafóricos. (Gómez, 2001: 306, la cursiva es nuestra).

- Asimismo, el uso de las formas españolas está sometido a «restricciones que tienen que ver con la norma estilística de repetición o redundancia de los textos españoles» (Gómez, 2001: 272). El cumplimiento de esta norma se consigue, por ejemplo, mediante la supresión de un adjetivo redundante o a través de la variación léxica.

Shaw (1979) realiza un estudio de compuestos técnicos motivados en alemán y en inglés, basándose en la observación de que la motivación para elegir una denominación puede ser distinta en dos lenguas y que éstas incluso pueden presentar tendencias denominativas diferentes. Después de un análisis sintáctico-semántico basado en casos, llega a algunas conclusiones generales sobre aspectos de motivación semántica, como por ejemplo (Shaw, 1979:194-195):

- Para el alemán, la de destacar el aspecto o la génesis del objeto.

- Para el inglés, la de destacar la función de una entidad o de describir «lo que es».

Nos parece un hallazgo muy interesante desde el punto de vista de la terminología y sería interesante poder contrastarlo con estudios de corpus en otros ámbitos temáticos y con pares de lenguas diferentes. 


\section{Conclusiones}

En este breve recorrido histórico hemos podido ver cómo las tendencias más importantes de la investigación lingüística de las distintas épocas y corrientes se reflejan en las aplicaciones que se han hecho al campo de la formación de palabras. En la gramática tradicional histórico-filológica, el énfasis se centra en la descripción diacrónica y etimológica, por una parte, y morfológica, por otra. Con la semántica estructural se ponen de relieve los aspectos semánticos de la palabra compuesta. En los enfoques generativos, en cambio, el interés central consiste en determinar las reglas que permiten la generación de compuestos. A partir del cambio de perspectiva pragmático-comunicativo, los enfoques pragmáticos y textuales abren una nueva perspectiva centrada en el uso. Estos enfoques han propiciado análisis de aspectos no contemplados anteriormente, como el de las distintas funciones de los compuestos. Los enfoques cognitivos, por otra parte, introducen otra perspectiva adicional al plantearse la representación mental de los compuestos y los mecanismos que intervienen en su interpretación. En los estudios basados en corpus de textos especializados, se identifican funciones específicas de los compuestos técnicos y se realiza una distinción entre compuestos con función denominativa y compuestos de construcción textual. Finalmente, de los resultados de los estudios contrastivos queremos destacar, sobre todo, la importancia de las relaciones semánticas y la posibilidad de divergencia, en este aspecto, de términos equivalentes en lenguas diferentes, debido a una motivación diferente, así como la identificación de factores textuales que pueden condicionar la elección del equivalente español para un compuesto alemán.

Los interrogantes que nos hemos planteado también dejan patente que la investigación de los compuestos nominales alemanes todavía tiene mucho que aportar al conocimiento del funcionamiento de la lengua. Como ejemplos concretos de vías de investigación posibles, podríamos mencionar:

- Desde el punto de vista de la terminología, las relaciones semánticas en compuestos especializados plantean cuestiones como las diferencias y similitudes entre las clasificaciones de compuestos generales y compuestos especializados, entre las clasificaciones de compuestos de ámbitos temáticos diferentes así como de lenguas diferentes ${ }^{6}$.

- En el campo del análisis del discurso especializado sería deseable una descripción más detallada de las funciones que desempeñan los compuestos en los textos especializados, especialmente aquellos que Zhu (1987) denomina compuestos de construcción textual.

- Desde un punto de vista contrastivo, una vez halladas las funciones textuales, se podría comparar cuáles son los mecanismos que desempeñan esas mismas funciones en los textos de otras lenguas, a través de un análisis contrastivo de corpus comparables ${ }^{7}$.

${ }^{6}$ Un primer intento en esta línea lo hemos realizado en Oster (2005).

7 Esta propuesta va en la dirección del estudio de Gómez (2001), aunque estamos pensando en corpus de textos originales en ambas lenguas, en vez de original y traducción. 
Vemos, pues, que el campo se amplía y nos muestra nuevas perspectivas que pueden ofrecer resultados interesantes en el futuro, sobre todo en lo que se refiere a los lenguajes especializados y la terminología, el aspecto textual y/o la comparación con otras lenguas. En palabras de Barz (2000: 310): «Der Wortbildungsforschung sind die Fragen nicht nur nicht ausgegangen, sondern es sind viele neue hinzugekommen.»

\section{REFERENCIAS BIBLIOGRÁFICAS}

Adelung, J. Chr. (1793-1801): Grammatisch-kritisches Wörterbuch der Hochdeutschen Mundart, Leipzig.

Anderson, Richard. C. y P. David PeARSON (1988): «A schema-theoretic view of basic processes in reading comprehension», Interactive Approaches to Second Language Reading, Patricia L. CARrell, et al. (eds), Cambridge, Cambridge University Press, pp. 37-55.

ANGele, Sybille. (1992): Nominalkomposita des Deutschen und ihre Entsprechungen im Spanischen. Eine kontrastive Untersuchung anhand von Texten aus Wirtschaft und Literatur, München, iudicium.

ARONOFF, Mark (1976): Morphological Investigations, University of Connecticut.

BARZ, Irmhild (2000): «Zum heutigen Erkenntnisinteresse germanistischer Wortbildungsforschung. Ein exemplarischer Bericht», Praxis - und Integrationsfelder der Wortbildungsforschung, Irmhild BARZ et al. (eds) Heidelberg, Winter, pp. 299-316.

BAUER, Laurie (1979): «On the need for pragmatics in the study of nominal compounding», Journal of Pragmatics (3), pp. 45-50.

Betten, Anne (1987): «Die Bedeutungen der Ad-hoc-Komposita im Werk von Thomas Bernhard», Neuere Forschungen zur Wortbildung und Historiographie der Linguistik, Brigitte AsBACH-SCHNITKER y Johannes RogGENHOFER (eds), Tübingen, Narr, pp. 69-90.

BREKLE, Herbert E. (ed.) (1983-1985): Kommunikative und pragmatisch-semantische Bedingungen der Aktualgenese, der Verwendung und des Verstehens von Nominalkomposita (edición en microficha), Regensburg.

Carroll, John M. y Michael Tanenhaus (1975): «Prolegomena to a Functional Theory of Word Formation», Papers from a Parasession on Functionalism, Robin E. GRossman et al. (eds), Chicago, Chicago Linguistic Society, pp. 47-62.

Cartagena, Nelson y Hans Martin Gauger (1989): Vergleichende Grammatik Spanisch-Deutsch, Mannheim, Dudenverlag.

COSERIU, Eugenio (1968): «Les structures lexématiques», Probleme der Semantik, W. Theodor Elwert (ed.), Wiesbaden, Steiner, pp. 3-16.

— (1977): «Inhaltliche Wortbildungslehre (am Beispiel des Typs «coupe-papier»)», Perpektiven der Wortbildungsforschung. Herbert E. BREKLE y Dieter KASTOVSKY (eds), Bonn, Bouvier, pp. 48-61.

DedERDING, Hans-Martin (1982): Wortbildung, Syntax, Text: Nominalkomposita und entsprechende syntaktische Strukturen in deutschen Patent- und Auslegeschriften, Erlangen, Palm \& Enke.

— (1983): «Wortbildung und Text. Zur Textfunktion (TF) von Nominalkomposita (NK)», Zeitschrift für germanistische Linguistik 13(1), pp. 49-64.

DoKuliL, Milos (1968): «Zur Theorie der Wortbildung.» Wissenschaftliche Zeitschrift der KarlMarx-Universität Leipzig 17, pp. 203-221.

DownING, Pamela (1977): «On the Creation and Use of English Compound Nouns», Language (53), pp. 810-842. 
DRESSLER, Wolfgang (1981): «Zum Verhältnis von Wortbildung und Textlinguistik (mit Beispielen aus der schönen Literatur)», Text vs. Sentence continued, Janos PETÖFI (ed.), Hamburg, Buske, pp. 96-106.

ERBEN, Johannes (1993, 2000): Einführung in die deutsche Wortbildungslehre, Berlin-Leipzig, E. Schmidt.

FAUCONNIER, Gilles (1985): Mental Spaces: Aspects of Meaning Construction in Natural Language, Cambridge, Massachusetts, Cambridge University Press.

FAUCONNIER, Gilles y Mark TuRner (1994): Conceptual Projection and Middle Spaces, Department of Cognitive Science. University of California, San Diego, pp. 1-39. [http://www.cogsci.ucsd. edu/research/files/technical/9401.pdf., fecha de consulta 04.11.05.]

FLEISCHER, Wolfgang $(1969,1975,1982)$ : Wortbildung der deutschen Gegenwartssprache, Tübingen, Niemeyer.

GAUGER, Hans-Martin (1971): Durchsichtige Wörter. Zur Theorie der Wortbildung, Heidelberg, Winter Universitätsverlag.

GÓMEZ PÉREZ, Carmen (2001): La composición nominal alemana desde la perspectiva textual: El compuesto nominal como dificultad de traducción del alemán al español, Tesis doctoral, Departamento de Traducción e Interpretación, Salamanca, Universidad de Salamanca.

GRIMM, Jacob (1878, primera edición 1826): Deutsche Grammatik, Gütersloh.

— (1890, primera edición 1831): Deutsche Grammatik, Gütersloh.

HENZEN, Walter (1957): Deutsche Wortbildung, Tübingen, Niemeyer.

HERINGER, Hans Jürgen (1984a): «Gebt endlich die Wortbildung frei!» Sprache und Literatur in Wissenschaft und Unterricht (15), pp. 43-53.

— (1984b): «Wortbildung: Sinn aus dem Chaos», Deutsche Sprache (12), pp. 1-13.

HERZOG, Reinhart (1976): «Gegenwärtige Tendenzen in der terminologischen Wortbildung. Untersuchungen zur Verwendung der Kompositumstruktur in Fachwörtern der amerikanischen und deutschen Datenverarbeitungsterminologie», Fachsprachen. Terminologie. Struktur. Normung, Karl-Heinz BAUSCH et al. (eds), Berlin, Beuth, pp. 73-79.

IsCHREYT, Heinz (1965): Studien zum Verhältnis von Sprache und Technik. Institutionelle Sprachlenkung in der Terminologie der Technik, Düsseldorf, Schwann.

JACKENDOFF, Ray (1975): «Morpological and Semantic Regularities in the Lexicon», Language (51), pp. 639-671.

KAUFFER, Maurice (2005): Les mots composés allemands en texte. Essai de synthèse méthodologique et critique, Frankfurt a. M., Peter Lang.

KüRSCHNER, Wilfried (1974): Zur syntaktischen Beschreibung deutscher Nominalkomposita. Auf der Grundlage generativer Transformationsgrammatiken, Tübingen, Niemeyer.

LEES, Robert B. (1960): The Grammar of English Nominalizations, Bloomington, Indiana University.

Marchand, Hans (1960, 1969): The Categories and Types of Present-Day English Word-Formation. A Synchronic-Diachronic Approach, München, Beck'sche Verlagsbuchhandlung.

MatusSEK, Magdalena (1994): Wortneubildung im Text. Hamburg, Buske.

Motsch, Wolfgang (1970): «Analyse von Komposita mit zwei Elementen», Progress in Linguistics: A collection of Papers, Manfred BIERWISch y Karl Erich HeIDOLPH (eds), Den Haag, Mouton, pp. 208-223.

OlsEN, Susan (1986): Wortbildung im Deutschen. Eine Einführung in die Theorie der Wortstruktur, Stuttgart, Alfred Körner Verlag.

ORTNER, Hanspeter y Lorelies ORTNER (1984): Zur Theorie und Praxis der Kompositaforschung, Tübingen, Narr.

ORTNER, Lorelies et al. (1991): Deutsche Wortbildung. Vierter Hauptteil: Substantivkomposita, Berlin, de Gruyter. 
OsTER, Ulrike (2005): Las relaciones semánticas de términos polilexemáticos. Estudio contrastivo alemán-español, Frankfurt a. M., Peter Lang.

PAUL, Hermann (1896): «Über die Aufgaben der Wortbildung», Sitzungsberichte der philosophischen und der historischen Classe der $k$. b. Akademie der Wissenschaften zu München, Jahrgang 1896, pp. 692-713.

— (1903): «Das Wesen der Wortzusammensetzung», Indogermanische Forschungen. Zeitschrift für Indogermanische Sprach—und Altertumskunde 14.

- (1920): Deutsche Grammatik, Halle (Saale).

PesCHEL, Corinna (2002): Zum Zusammenhang von Wortneubildung und Textkonstitution, Tübingen, Niemeyer.

RICKHEIT, Mechthild (1993): Wortbildung: Grundlagen einer kognitiven Wortsemantik, Opladen, Westdeutscher Verlag.

RYDER, Mary E. (1994): Ordered Chaos: The Interpretation of English Noun-Noun-Compounds, San Diego, University of California Press.

SCHRÖDER, Marianne (1978): «Über textverflechtende Wortbildungselemente», Deutsch als Fremdsprache, 78/2, pp. 85-92.

— (1983): «Zum Anteil von Wortbildungskonstruktionen an der Konstitution von Texten», Beiträge zur Erforschung der deutschen Sprache (Beiträge zur Geschichte der deutschen Sprache und Literatur) (3), pp. 108-119.

— (1985): «Überlegungen zur textorientierten Wortbildung», Linguistische Studien, Reihe A, Arbeitsberichte. Textbezogene Nominationsfoschung. Studien zur deutschen Gegenwartssprache, Berlin, Akademie der Wissenschaften der DDR, Zentralinstitut für Sprachwissenschaften, pp. 69-94.

SHaw, J. Howard (1979): Motivierte Komposita in der deutschen und englischen Gegenwartssprache, Tübingen, Narr.

STÖHR, Ingo (1984a): «Pragmatik und Nominalkomposition. Arbeitsbericht 40», Kommunikative und pragmatisch-semantische Bedingungen der Aktualgenese von Nominalkomposita. 19831985 (edición en microficha), Herbert E. BREKLE (ed.), Regensburg, Universität Regensburg.

- (1984b): «Zusammenfassende Darstellung der experimentellen Ergebnisse zur ad-hoc Nominalkomposition. Arbeitsbericht 39», Kommunikative und pragmatisch-semantische Bedingungen der Aktualgenese von Nominalkomposita. 1983-1985 (edición en microficha). Herbert E. BREKLE. (ed.), Regensburg, Universität Regensburg, pp. 1-29.

TOMAN, Jindrich (1983): Wortsyntax, Tübingen, Niemeyer.

TURNER, Mark y Gilles FAUCONNIER (1995): «Conceptual Integration and Formal Expression», Metaphor and Symbolic Activity 10(3), pp. 183-204.

WILDGEN, Wolfgang (1982): «Makroprozesse bei der Verwendung nominaler Ad-hoc-Komposita im Deutschen», Deutsche Sprache, pp. 237-257.

Wilmans, W. (1896):. Deutsche Grammatik, zweite Abteilung: Wortbildung, Berlin-Leipzig.

Wladowa, E. W. (1975): «Okkasionelle Wortbildungen mit dem gleichen Stamm als Satz- und Textverflechtungsmittel (nachgewiesen an E. Strittmatters «Ole Bienkopp», Aufbau-Verlag, Berlin 1963)», Textlinguistik (4), pp. 71-87.

Wolf, Barbara (1990): Nominalkompositionen im Deutschen und Französischen. Eine Untersuchung der französischen Entsprechungen zu deutschen Nominalkomposita aus verschiedenen fachsprachlichen Sachbereichen, Münster, Kleinheinrich.

ZHU, Jianhua (1987): Morphologie, Semantik und Funktion fachsprachlicher Komposita. Analyse von Fachtexten der Silikattechnik, Heidelberg, Groos.

- (1990): «Fachsprachliche Komposita in pragmatischer und textueller Sicht», Dialog. Festschrift für Siegfried Grosse, Gert RICKHEIT y Sigurd WICHTER (eds), Tübingen, Niemeyer, pp. 221-232. 